\title{
Escherichia coli damages host DNA
}

Common gut bacteria can induce eukaryotic chromosomal instability in vivo, according to a study published in a recent issue of the Proceedings of the National Academy of Sciences USA.

Up to $34 \%$ of commensal Escherichia coli strains isolated from human faeces in high-income countries contain $p k s$, a genomic island encoding a cluster of nonribosomal peptide synthetases and polyketide synthetases that are involved in the production of the genotoxin colibactin. This toxin had previously been shown to damage the DNA of eukaryotic cells in vitro.

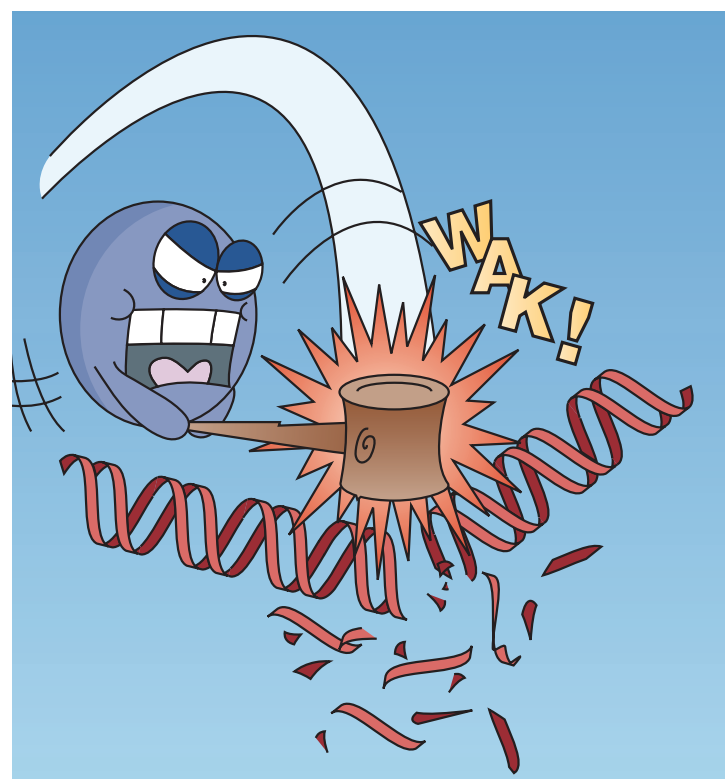

Now, Cuevas-Ramos and colleagues have found evidence that this toxin also causes DNA damage in vivo, providing a possible link between the intestinal flora and colon cancer.

Using a ligated colon model in mice, the authors showed that the promoter of $c l b A$, which encodes an essential enzyme in the colibactin synthesis pathway, was active when E. coli was in the intestine and that the bacterium was in close apposition to the intestinal brush border. To determine whether the toxin-expressing bacteria induced damage to the host DNA in vivo, the authors investigated the phosphorylation state of Ser139 in histone H2AX (known as $\gamma$-H2AX when phosphorylated), which is a marker for double-strand breaks in the chromosome. Cells exposed to pks-containing E. coli had three times as many $\mathrm{H} 2 \mathrm{AX}$ foci and twice as much $\gamma$-H2AX as cells exposed to $c l b A$ mutants or untreated controls, and were similar in this respect to tissue that had been exposed to 0.5 grays of ionizing radiation.

The authors then determined the consequences of the double-strand breaks using an in vitro model. First, they showed that more Chinese hamster ovary $(\mathrm{CHO})$ cells were arrested at the G2-M stage of the cell cycle after exposure to $p k s$ containing E. coli than after exposure to $E$. coli lacking $p k s$, indicating a transient activation of the DNA damage response. In $\mathrm{CHO}$ cells that were deficient in the repair of double-strand breaks, pks-containing E. coli induced a large increase in the number of apoptotic cells compared with numbers in cells exposed to E. coli lacking $p k s$ and in untreated cells. Looking at the chromosomes in more detail, the authors discovered that pks-containing E. coli induced the formation of anaphase bridges (the lingering DNA connections between misrepaired chromosomes that are pulled between anaphase poles) and nucleoplasmic bridges (connections between separated sister cells that are formed by anaphase bridges). Furthermore, pks-containing E. coli induced aneuploidy, which is a common consequence of anaphase bridges. Finally, the authors demonstrated that, in addition to causing chromosome abnormalities, exposure to pks-containing E. coli increased the level of mutations in host cells.

Together, these results indicate that certain strains of E. coli can induce chromosome instability and mutations in host cells and may therefore be contributing factors to colon cancer.

Christiaan van Ooij

ORIGINAL RESEARCH PAPER Cuevas-Ramos, G et al. Escherichia coli induces DNA damage in vivo and trigers instability in mammalian cells. Proc. Natl Acad. Sci. USA 107, 11537-11542 (2010) 